\title{
Nanodelivery of Triamcinolone Acetonide using PLGA-Chitosan Nanoparticles for the Treatment of Inflammation-related Ocular Cataract
}

\section{Yi Xing}

Zhengzhou University First Affiliated Hospital

\section{Lijuan Zhu}

Zhengzhou University First Affiliated Hospital

\section{Ke Zhang}

Zhengzhou University First Affiliated Hospital

\section{Teng Li}

Zhengzhou University First Affiliated Hospital

Shaohua Huang ( $\sim$ SpencerNewmanxMsMn@yahoo.com )

Zhengzhou University First Affiliated Hospital

\section{Nano Express}

Keywords: Cataract, PLGA, Chitosan, nanoparticles, inflammation, ocular disease

Posted Date: March 4th, 2020

DOl: https://doi.org/10.21203/rs.3.rs-15985/v1

License: (c) (1) This work is licensed under a Creative Commons Attribution 4.0 International License. Read Full License 


\section{Abstract}

This preprint has been removed from Research Square. 